\title{
Performance of a 3D convolutional neural network in the detection of hypoperfusion at CT pulmonary angiography in patients with chronic pulmonary embolism: a feasibility study
}

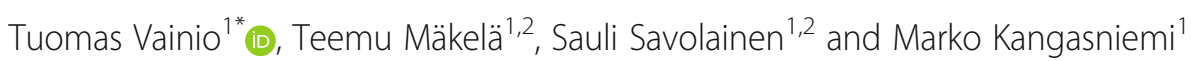

\begin{abstract}
Background: Chronic pulmonary embolism (CPE) is a life-threatening disease easily misdiagnosed on computed tomography. We investigated a three-dimensional convolutional neural network (CNN) algorithm for detecting hypoperfusion in CPE from computed tomography pulmonary angiography (CTPA).

Methods: Preoperative CTPA of 25 patients with CPE and 25 without pulmonary embolism were selected. We applied a 48\%-12\%-40\% training-validation-testing split (12 positive and 12 negative CTPA volumes for training, 3 positives and 3 negatives for validation, 10 positives and 10 negatives for testing). The median number of axial images per CTPA was 335 (min-max, 111-570). Expert manual segmentations were used as training and testing targets. The CNN output was compared to a method in which a Hounsfield unit (HU) threshold was used to detect hypoperfusion. Receiver operating characteristic area under the curve (AUC) and Matthew correlation coefficient (MCC) were calculated with their 95\% confidence interval (Cl).

Results: The predicted segmentations of CNN showed AUC 0.87 ( $95 \% \mathrm{Cl} 0.82-0.91$ ), those of HU-threshold method 0.79 (95\% Cl 0.74-0.84). The optimal global threshold values were CNN output probability $\geq 0.37$ and $\leq-850 \mathrm{HU}$. Using these values, MCC was 0.46 (95\% Cl 0.29-0.59) for CNN and 0.35 (95\% Cl 0.18-0.48) for HU-threshold method (average difference in MCC in the bootstrap samples 0.11 (95\% Cl 0.05-0.16). A high CNN prediction probability was a strong predictor of CPE.
\end{abstract}

Conclusions: We proposed a deep learning method for detecting hypoperfusion in CPE from CTPA. This model may help evaluating disease extent and supporting treatment planning.

Keywords: Computed tomography angiography, Deep learning, Feasibility Studies, Neural networks (computer), Pulmonary embolism

\footnotetext{
* Correspondence: tuomas.j.vainio@helsinki.fi; tuomas.vainio@hus.fi

'HUS Medical Imaging Center, Radiology, University of Helsinki and Helsinki University Hospital, P.O. Box 340 (Haartmaninkatu 4), Fl-00290 Helsinki,

Finland

Full list of author information is available at the end of the article
}

() The Author(s). 2021 Open Access This article is licensed under a Creative Commons Attribution 4.0 International License, which permits use, sharing, adaptation, distribution and reproduction in any medium or format, as long as you give appropriate credit to the original author(s) and the source, provide a link to the Creative Commons licence, and indicate if changes were made. The images or other third party material in this article are included in the article's Creative Commons licence, unless indicated otherwise in a credit line to the material. If material is not included in the article's Creative Commons licence and your intended use is not permitted by statutory regulation or exceeds the permitted use, you will need to obtain permission directly from the copyright holder. To view a copy of this licence, visit http://creativecommons.org/licenses/by/4.0/. 


\section{Key points}

- The study aim was to detect hypoperfusion in chronic pulmonary embolism.

- Convolutional neural network was applied to computed tomography pulmonary angiography images.

- Good accuracy with moderate segmentation correlation to manual delineations was achieved.

- The model offered an improvement compared to a computed tomography density-based analysis.

\section{Background}

Chronic thromboembolic pulmonary hypertension (CTEPH) is a late complication of pulmonary embolism $[1,2]$. Unresolved thrombus is seen in $15-30 \%$ of patients 9-12 months after the initial pulmonary embolism $[3,4]$, which may organise and progress into chronic thromboembolic disease (CTED) [5]. Pulmonary vasculopathy may occur in the nonoccluded arteries [6]. Consequently, pulmonary vascular resistance and arterial pressure increase developing CTEPH [7] with a cumulative incidence of $0.1-9.1 \%$ within 2 years after a symptomatic pulmonary embolism event [8]. The incidence of CTED is estimated to be significantly higher [9]. Unlike other forms of pulmonary hypertension (PH), CTEP $\mathrm{H}$ is potentially curable surgically with pulmonary endarterectomy $[10,11]$. If untreated, the median survival is less than 2 years if the mean pulmonary arterial pressure is $>30 \mathrm{mmHg}[1,2]$. Hence, early diagnosis is cardinal for these patients.

The screening method of choice for CTEPH is ventilation/perfusion (V/Q) scan [12] while catheter-directed pulmonary angiography is considered the reference standard in confirming the diagnosis $[13,14]$. Computed tomography pulmonary angiography (CTPA) can also be used to confirm the diagnosis of CTEPH [15-17] and estimate the severity of the pulmonary hypertension $[18$, 19]. Despite its low sensitivity in clot detection in the distal and subsegmental vessels [20], CTPA is fundamental in the diagnostic workup of CTEPH with a crucial role in assessing the feasibility of surgical treatment [17]. CTPA is noninvasive and more widely available than catheter-directed pulmonary angiography and has the advantage of evaluating the parenchymal and bronchial signs, the collateral circulation, and competing diagnoses [21].

In CTPA, the vascular signs for chronic pulmonary embolism (CPE) are complete occlusion of the pulmonary arteries or incomplete occlusion by nonobstructing bands, webs, or laminated thrombi [5, 21]. A typical parenchymal sign for CPE is mosaic perfusion, seen as welldemarcated regions of hypoattenuation representing hypoperfusion and hyperattenuating regions representing the blood flow to the patent pulmonary vascular bed [5, $21]$. These radiological signs are frequently missed in the reports [22], and because of the disease insidious nature and the unspecific clinical presentation [23], the diagnosis is often delayed, with a median of 14 months after the initial symptoms [24]. A high index of suspicion is necessary for the diagnosis [21].

Several techniques have been developed for computeraided detection and diagnosis of pulmonary embolism $[25,26]$. However, no studies have been done on artificial intelligence techniques to detect parenchymal changes from CTPA relating to hypoperfusion in CPE. An automatic tool detecting these perfusion defects from CTPA images could potentially aid in diagnostics, evaluating the extent of the disease, and treatment planning of chronic pulmonary embolism. This study aimed to train a convolutional neural network $(\mathrm{CNN})$ to detect the hypoperfused regions affected by CPE from CTPA images and evaluate the algorithm performance in this task.

\section{Methods}

\section{Subjects and imaging parameters}

We retrospectively reviewed reports of V/Q scans performed in Helsinki University hospital between January 2017 and December 2019. Based on the reports, 30 patients with findings suggestive of CPE in the V/Q scan were selected for the study. Also, 31 patients with no signs of pulmonary embolism in the V/Q scan were selected.

The inclusion criteria for the positive cases were a positive V/Q scan for CPE and a CTPA with signs of CPE performed in our hospital district within 3 months before or after the positive V/Q scan before treatment without signs of acute pulmonary embolism. Cases with no possibility to delineate areas of hypoperfusion at CTPA were excluded from the study. The negative patients' inclusion criteria were a negative V/Q scan for acute or chronic pulmonary embolism and a negative CTPA for acute or chronic pulmonary embolism performed within three months of the negative V/Q scan. In both groups, CTPA studies, in which the radiological signs of a parenchymal disease unrelated to CPE (e.g., hyperattenuation caused by talcosis) extended over two thirds of the lung volume, were excluded. Artefacts caused by foreign material that covered more than one third of the lung volume in the CTPA was a criterion for exclusion in both groups.

Five patients with positive findings were excluded from the study for the lack of presurgical CTPA in the picture archive, a lack of visible parenchymal hypoattenuation in the CTPA, lungs completely affected by CPE on the V/Q scan without demarcating difference of attenuation at CTPA, no CTED or CTEPH diagnosed clinically, and 
inclusion of a massive acute or chronic thromboembolism. Six patients with CPE-negative findings were excluded based on too large slice thickness on CTPA in our picture archive, parenchymal lesions covering over two thirds of the lung volume related to another disease (talcosis), acute pulmonary embolism, a suspicion of CPE at CTPA (two patients), and significant artefacts on CTPA related to prior surgery. After the exclusion, 25 positive (group A) and 25 negative (group B) cases were included in the study. Patient demographics are shown in Table 1.

In group A, 18 patients had a clinical diagnosis of CTEPH, clinically defined as the mean pulmonary arterial pressure $>25 \mathrm{mmHg}$ at rest, pulmonary capillary wedge pressure $<15 \mathrm{mmHg}$ documented with a right heart catheterisation, persisting mismatched perfusion defect in V/Q scan with specific diagnostic signs for CTEPH on CTPA or conventional pulmonary angiography [27]. Seven patients had CTED, defined with the same criteria as mentioned above, excluding pulmonary hypertension [28]. Seventeen patients with CTEPH had pulmonary hypertension confirmed with a right heart catheterisation. One CTEPH patient had only an echocardiogram done for the diagnosis. A normal pulmonary arterial pressure was confirmed with a right heart catheterisation for one of the patients with CTED. The rest did not warrant a right heart catheterisation.

The CTPA studies were performed before treatment on various scanners in multiple hospitals within the Hospital District of Helsinki and Uusimaa. The protocol was defined by the joint municipal authority for specialised healthcare. Different tube voltages were used depending on the scanner and the size of the patient (see Table 2 for details). All data were anonymised and stored on a server running the Extensible Neuroimaging Archive Toolkit version 1.1.6 [29].

\section{Preprocessing}

A radiologist with over 5 years of experience manually segmented the hypoperfused regions on the CTPA images for the 25 patients in group A, using V/Q scan images as a reference for segmentation. To appreciate the minor differences in the parenchymal attenuation, greylevel mapping with a window width ranging from 200 to 900 and a window level ranging from $-1,100$ to -800 was used. Only the regions of hypoperfusion visible in the
CTPA were segmented. Regions where hypoattenuation was suspectedly caused by lung disease other than CPE were not segmented. Areas with relevant artefacts relating to, e.g., motion or beam hardening, were left unsegmented. Regions smaller than $5 \mathrm{~mL}$ were left unsegmented. Hyperattenuating lesions relating to the CPE were included in the segmentation, e.g., lung infarctions and atelectasis in the diseased area if seen in the hypoperfused region. Segmentation was done on the axial images using the image processing and visualisation platform 3D Slicer [30].

Due to a large number of axial images, with a median of 335 (min-max, 111-570) per CTPA, we used a tool based on morphological contour interpolation for assistance in segmentation [31], which allowed any number of slices to be skipped between slices segmented manually. This tool interpolates the manually segmented slices by detecting and aligning overlapping shapes in the adjacent segmented slices and generating a transition sequence of one-pixel dilatation between the overlapping shapes, and a median of this sequence is taken as a result to fill the skipped slices [31, 32]. The radiologist segmented the hypoperfused regions to every $3-10$ axial images by hand, and the complete segmentation was created by the interpolation tool. Contours of the interpolation were evaluated and approved by the radiologist before committing to the segmentation. If the radiologist disagreed with the interpolation, the contours of the interpolated segmentation were modified and corrected manually. In group A, segmentation was done in this manner to every axial CTPA image for the CNN training and analysis. In group $\mathrm{B}$, there was no manual segmentation done for hypoperfusion. Additionally, in both groups, the lungs were segmented as a whole, and the CTPA image data outside of the lungs was excluded from the analysis.

We applied a $48 \%-12 \%-40 \%$ training-validation-test split, i.e., 12 CTEPH positive and 12 negative CTPA volumes for training, 3 positives and 3 negatives for validation, 10 positives, and 10 negatives for testing. We split the data temporally: all the training and validation data had been imaged before all the test data. The hospital ethics committee approved the study. Informed consent was waived because of the retrospective design and anonymous clinical data used for the analysis.

Table 1 Patient demographics

\begin{tabular}{|c|c|c|c|c|c|}
\hline & Median age (years, min-max) & Males & Females & CTEPH patients & CTED patients \\
\hline Group A & $67(21-82)$ & 8 & 17 & 18 & 7 \\
\hline Group B & $67(33-88)$ & 11 & 14 & 0 & 0 \\
\hline All & $67(21-88)$ & 19 & 31 & 18 & 7 \\
\hline
\end{tabular}

CTEPH Chronic thromboembolic hypertension, CTED Chronic thromboembolic disease 
Table 2 Number of patients imaged with each computed tomography scanner and tube voltage

\begin{tabular}{|c|c|c|c|c|c|}
\hline \multirow[b]{2}{*}{ Scanner model (manufacturer) } & \multicolumn{4}{|c|}{ Tube voltage } & \multirow[b]{2}{*}{ Total } \\
\hline & $80 \mathrm{kV}$ & $100 \mathrm{kV}$ & $120 \mathrm{kV}$ & $140 \mathrm{kV}$ & \\
\hline Somatom Definition AS (Siemens Healthineers) & 0 & 2 & 1 & 0 & 3 \\
\hline Discovery HD (General Electric Healthcare) & 0 & 4 & 0 & 0 & 4 \\
\hline Somatom Definition Edge (Siemens Healthineers) & 1 & 6 & 1 & 2 & 10 \\
\hline Somatom Definition Flash (Siemens Healthineers) & 0 & 9 & 2 & 0 & 11 \\
\hline Lightspeed VCT (General Electric Healthcare) & 0 & 9 & 1 & 0 & 10 \\
\hline Aquilon Prime (Toshiba) & 0 & 1 & 0 & 0 & 1 \\
\hline Revolution EVO (General Electric Healthcare) & 0 & 2 & 2 & 0 & 4 \\
\hline Revolution HD (General Electric Healthcare) & 1 & 5 & 0 & 1 & 7 \\
\hline Total & 2 & 38 & 7 & 3 & 50 \\
\hline
\end{tabular}

Patients were imaged with one of the eight scanner models depending on the referring hospital. Tube voltages ranged from $80 \mathrm{kV}$ to $140 \mathrm{kV}$

\section{Neural network training}

We trained a 12 layers deep U-net-type [33] $\mathrm{CNN}$ with three-dimensional convolutional layers $(3 \times 3 \times 3$ kernel size; valid padding; 16,32 , or 64 filters each), three maxpooling/upsampling steps with skip connections, and a single output neuron with sigmoid activation (see details in Fig. 1). Exponential linear units were used as activations in the hidden layers. The $\mathrm{CNN}$ was implemented using Keras in Tensorflow framework version 2.0 [34]. The compute tomography (CT) angiography volumes were resampled to uniform $1 \times 1 \times 1 \mathrm{~mm}^{3}$ and normalised by linearly shifting and scaling the $-900 \ldots-600 \mathrm{HU}$ range into $-1 \ldots 1$ range. The training data were divided into $32 \times 32 \times 32$ voxel patches, and the patches completely outside the lung region were rejected. These samples with borders extended by 30 voxels on each side (due to a valid padding scheme in all the convolutional layers) were fed into the network with batch size 16 . During training, Adam optimiser [35] with $2 \times 10^{-4}$ learning rate and Dice loss [36] against manual labels were used. The final model was achieved after 21 epochs, at which point the validation accuracy stopped improving. Also, no further improvement on validation data was achieved by lowering the learning rate after the 21st epoch.

\section{Performance and statistical testing}

We compared the CNN model to a method which used a simple cutoff Hounsfield units (HU) threshold: voxels inside the lungs with a $\mathrm{HU}$ value below a specific threshold value were labelled as hypoattenuation. Hereafter, we will refer to this global threshold approach as the "naive HU-threshold method", in contrast to an active expert-selected threshold for each patient or region. We calculated the voxel segmentation receiver operating characteristic curves for these two methods and compared the area under curve (AUC) values produced by the $\mathrm{CNN}$ model and the naïve HU-threshold method.

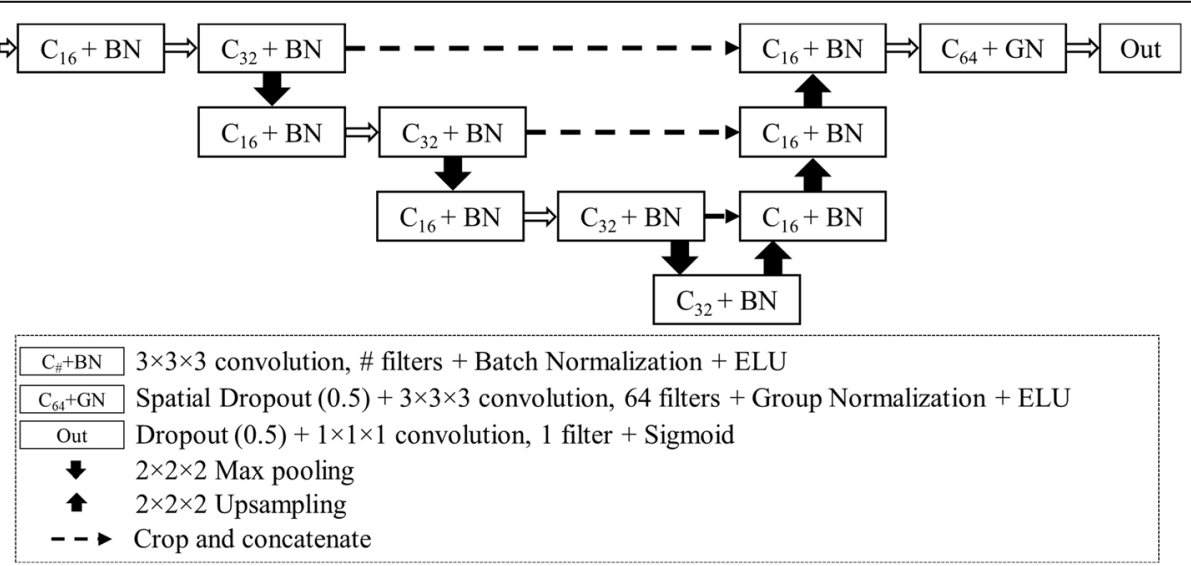

Fig. 1 The convolutional neural network architecture was based on U-net with three max-pooling steps in the downsampling pathway and linear upsampling in the resolution recovery path. Convolutional layers (C) were followed by batch or group normalisation and exponential linear unit (ELU) activations. Skip connections with cropping were used between the matching resolution levels (dashed arrows). C Convolutional layer, BN Batch normalisation, ELU Exponential linear unit, GN Group normalisation 
Due to inherent class imbalance in the dataset $(21 \%$ of lung voxels in the total dataset were labelled as hypoperfused), we chose the Matthews correlation coefficient (MCC) [37] as the segmentation performance metric. Secondly, in contrast to the more commonly used $F_{1}$ metric, which emphasises the positive class, MCC remains agnostic of which of the binary classes is selected "positive". Therefore, we avoid imposing hypoattenuating areas to be more important than the remaining tissues in the segmentation performance evaluation. Confidence intervals (CIs) for the reported performance metrics were calculated using a bootstrap method by resampling with replacement with $10^{5}$ repeats and 95\% significance level. The optimal threshold for visual inspections was chosen by maximising the test set balanced accuracy. We evaluated the test cases visually to assess the clinical relevance of the false positive and negative segmentations. We further investigated if the $\mathrm{CNN}$ probability or the mean $\mathrm{HU}$ could indicate the presence of the CPE.

\section{Results}

\section{Segmentation performance}

An example of manual, naïve $\mathrm{HU}$-threshold and $\mathrm{CNN}$ segmentations is shown in Fig. 2. Time for manual segmentation was approximately $5 \mathrm{~h}$ per case.

Using the naive $\mathrm{HU}$-threshold method over all the test samples, the ROC AUC was 0.79 (95\% CI 0.74-0.84); using the CNN model the ROC AUC was 0.87 (0.820.91) (see Fig. 3). The optimal global threshold value was $\leq-850 \mathrm{HU}$ for the threshold method and CNN output probability $\geq 0.37$. We used these values in the following MCC and visual assessments. MCCs were 0.35 (95\% CI $0.18-0.48$ ) and 0.46 (95\% CI $0.29-0.59$ ), for the naïve HU-threshold method and the CNN model, respectively. The average difference in the MCCs between the two methods in the bootstrap samples was 0.11 (95\% CI 0.05-0.16; positive value meaning $\mathrm{CNN}$ performed better). Because the $95 \% \mathrm{CI}$ excluded zero, we conclude in favour of a statistically significant improvement achieved by using the CNN model.

\section{Visual assessment}

Complementary to voxel-wise performance evaluation, we reviewed each test set CTPA by visually assessing individual regions segmented by the $\mathrm{CNN}$ model and the naïve HU-threshold method (Fig. 4). Regions overlapping the manually segmented areas were defined as true positives. Regions not overlapping with the manually segmented areas were defined as false positives. Manually segmented regions not overlapping with the segmentation done by the prediction algorithm were defined as false negatives. If more than one part segmented by the prediction algorithm overlapped a same manually segmented region, they were all defined together as one true positive. We ignored prediction labels outside the lung parenchyma.

In the $\mathrm{CNN}$ output, there were 63 independent false positive labels in group $A$ and 111 false positive labels in group B, respectively. Beam hardening artefacts from the larger organs, including the heart, the hilum of the lung, the aorta, and the diaphragm, were seen in $17 \%$ of the false positives. In $16 \%$, there were beam hardening artefacts relating to the contrast material bolus in either superior vena cava, subclavian, or brachiocephalic veins. Thirty-three per cent of the false positives were adjacent to smaller blood vessels and 15\% were minor subpleural regions most likely related to beam hardening (Fig. 5).

Twelve areas of varying sizes were labelled positive by the $\mathrm{CNN}$, which were incorrectly labelled negative in the preprocessing. Nine per cent of the false positives remained unspecific by nature. In one case, there was air trapping relating to a mucus plug in a bronchus. One patient in group B had gone through left upper lobe pneumonectomy in the past, and the majority of the remaining lung volume was detected as false positive by the $\mathrm{CNN}$ algorithm and HU-threshold method (false positive outlier, Fig. 6b; Fig. 7).

Of the 137 manually segmented lesions, 133 were correctly labelled positive by the CNN. There were both overestimation and underestimation of these lesions. The overestimation of 8 true positive labels was related to hypoattenuation caused by beam hardening artefacts. Otherwise, the cause of the overestimation and underestimation remained unspecific.

In the naïve $\mathrm{HU}$ threshold algorithm, each voxel below the certain threshold was labelled as positive. With the optimal HU-threshold of $<-850$, the result consisted of numerous voxel clouds of different sizes, including single voxels, because no voxel clustering was used. As this method labelled a myriad of regions positive, ranging from one voxel size to larger areas, it was not reasonable to count them. Hence, we present the visual assessment of the naïve HU-threshold method descriptively.

The naive $\mathrm{HU}$ threshold algorithm responded to general noise in the lung parenchyma consisting of both morphologic variegation and imaging noise, which can be seen in the healthy and disease-associated lung parenchyma. Hypoattenuating voxels caused by artefacts were also marked as false positives. Each manually segmented hypoperfusion area contained at least one voxel labelled as true positive. As shown in Fig. 6, both the mean over the lung tissue with $<-850 \mathrm{HU}$ and the average CNN prediction probability, i.e., network's confidence in the voxel segmentation, indicate the presence of CPE. Using the $\mathrm{CNN}$ and value 0.55 (dashed line in Fig. $6 \mathrm{~b}$ ), all but one test case was correctly categorised. The network inference time of the $\mathrm{CNN}$ model for a single CTPA in 

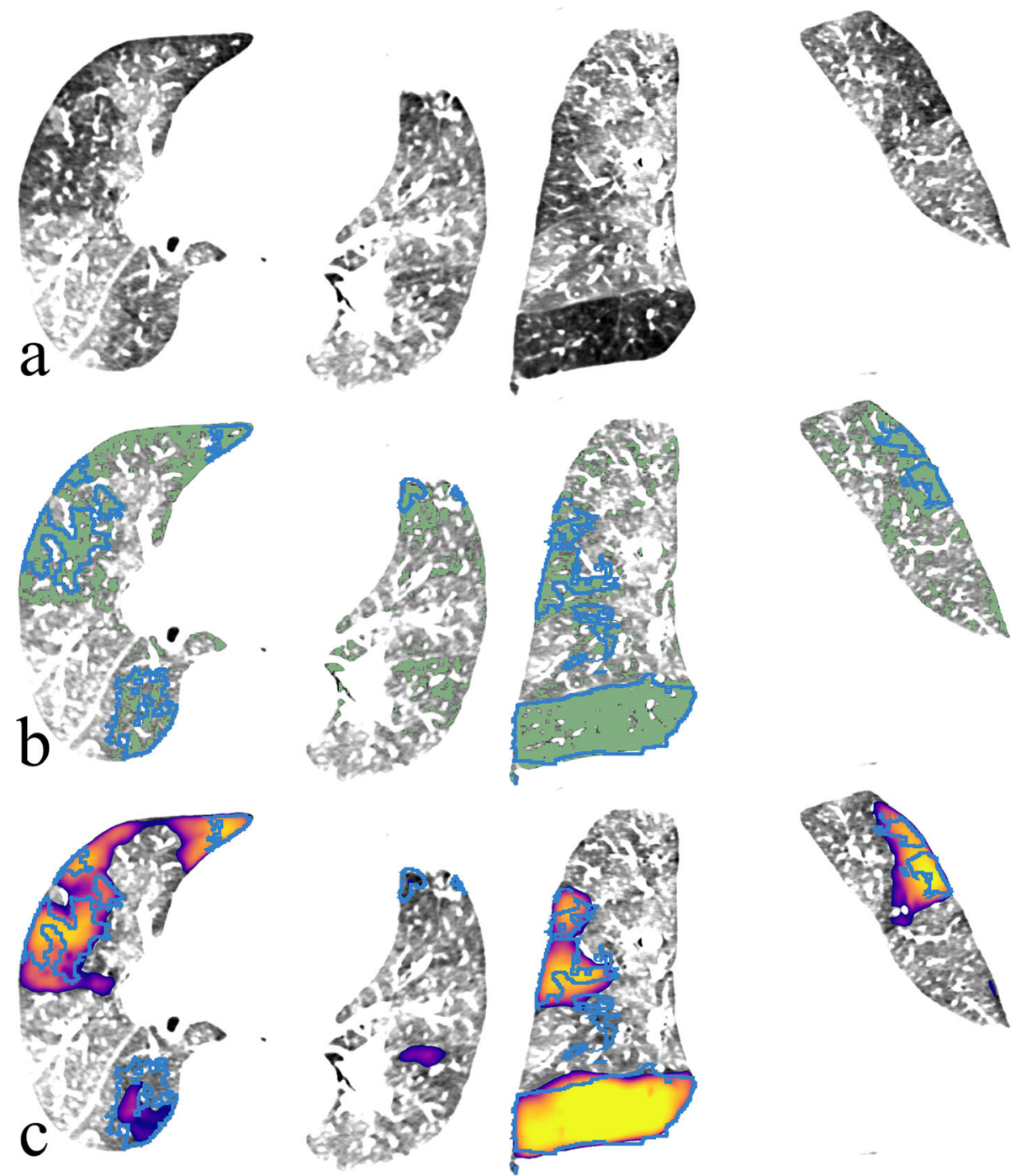

Fig. 2 An example of computed tomography angiography volume (a) segmentations with good agreement to manual delineations (blue outline): HU threshold ( $\leq-850)$-derived segmentation is shown in green $(\mathbf{b})$, while the prediction of convolutional neural network is shown as colourmap ( $\geq 0.37$ probability cutoff) (c). HU Hounsfield units

the test set was between 17 and $32 \mathrm{~s}$ using an NVIDIA Quadro M6000 24GB graphics card.

\section{Discussion}

As far as we know, this is the first study to evaluate the feasibility of three-dimensional CNN in the detection of lung hypoperfusion from CTPA images in CPE. Our CNN algorithm showed an encouraging performance in detecting these lesions on a separate testing set. Since there are no previous studies to compare our results to, we compared the $\mathrm{CNN}$ algorithm to a method based on a simple HU cutoff threshold. Using even the HU threshold segmentation could help visual evaluation, but according to our results, the $\mathrm{CNN}$ algorithm could offer a significant improvement with a 0.87 ROC-AUC and
$0.46 \mathrm{MCC}$ compared to the 0.79 and 0.35 for the naïve HU-threshold method, respectively.

The average $\mathrm{CNN}$ prediction probability indicates the presence of $\mathrm{CPE}$, and all but one of the $\mathrm{CPE}$ and control cases in the test set were correctly categorised using a probability value 0.55 . Therefore, we propose that in addition to the lesion segmentation, the disease overall presence could be inferred using a CNN-based approach.

The CNN algorithm recognised most (97\%) of the manually segmented lesions. Majority (82\%) of the false positive CNN labels were small and related to beam hardening artefacts from dense organs, which seemed to be the most challenging for the $\mathrm{CNN}$ to differentiate from true positive lesions. However, one patient had 


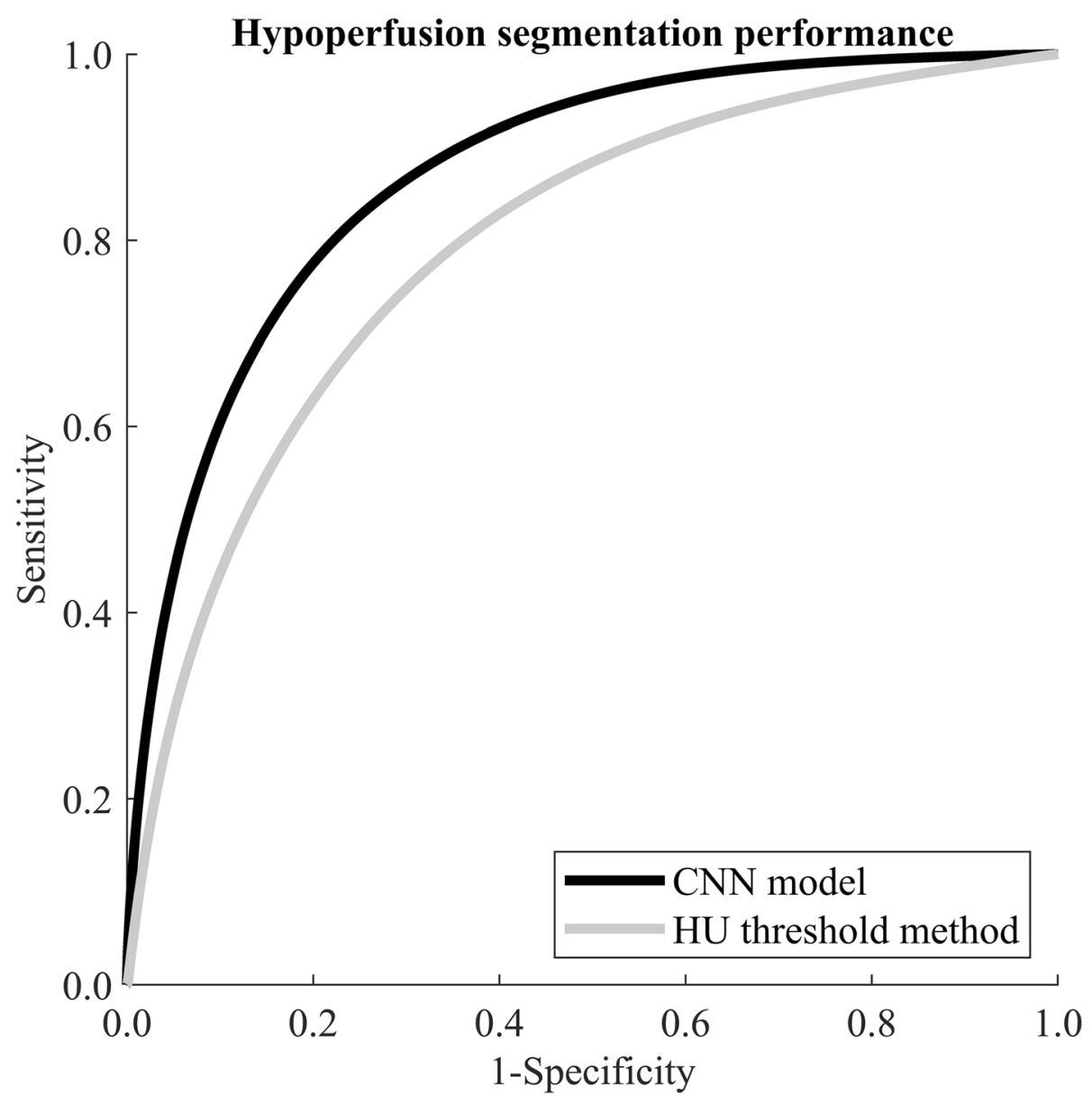

Fig 3 Receiver operating characteristic curves when applying the convolutional neural network (CNN) model or using a global naïve Hounsfield unit threshold method. The areas under the curves were 0.87 and 0.79 , respectively. HU Hounsfield units

extensive false positive labels covering most of the lung volume. This patient had undergone left upper lobe pneumonectomy and mean HU of the lung parenchyma was one of the lowest in the study group. After lobectomy, the remaining lung has a relative increase of air and a decrease of the lung parenchyma in the thorax seen as hyperlucency on a CT image [38]. This is likely the reason for the hyperlucency in this patient's lung and the $\mathrm{CNN}$ false positive labelling.

There was both overestimation and underestimation in the sizes of the hypoperfused lesions. The overestimation was partly related to the beam hardening artefacts mentioned earlier, but in most cases, the overestimation and underestimation remained unspecific. Since accurate delineation of the hypoattenuating regions in the CTPA was challenging, we had to compromise the manual segmentation in cases where the borders between affected and unaffected areas were gradient. This might partially explain the overestimation and underestimation of the lesion sizes in the labels output by the $\mathrm{CNN}$ algorithm.
CNNs have been previously applied in pulmonary embolism detection based on labelling the occluding clots seen in CTPA as filling defects of intravascular contrast material in pulmonary arteries [39-42]. These studies have focused mainly on acute pulmonary embolism. We took a different approach, and instead of the actual vascular defects, we focused on the hypoattenuation in the lung parenchyma relating to the hypoperfusion caused by chronic pulmonary embolism. Öman et al. (2019) have successfully implemented this type of approach to detect acute stroke lesions from CT angiography images with a CNN based algorithm [43]. However, since the lungs are composed largely of air, the differences in parenchymal enhancement are subtler between the hypoand hyperperfused regions at CTPA than at CT angiography of the brain. Another significant difference in the setting is that a single thrombus usually causes the ischemic stroke, whereas chronic pulmonary embolism affects many branches of the pulmonary vasculature by various degrees of obstruction. Unlike the brain, the attenuation of the lung is sensitive to regional 


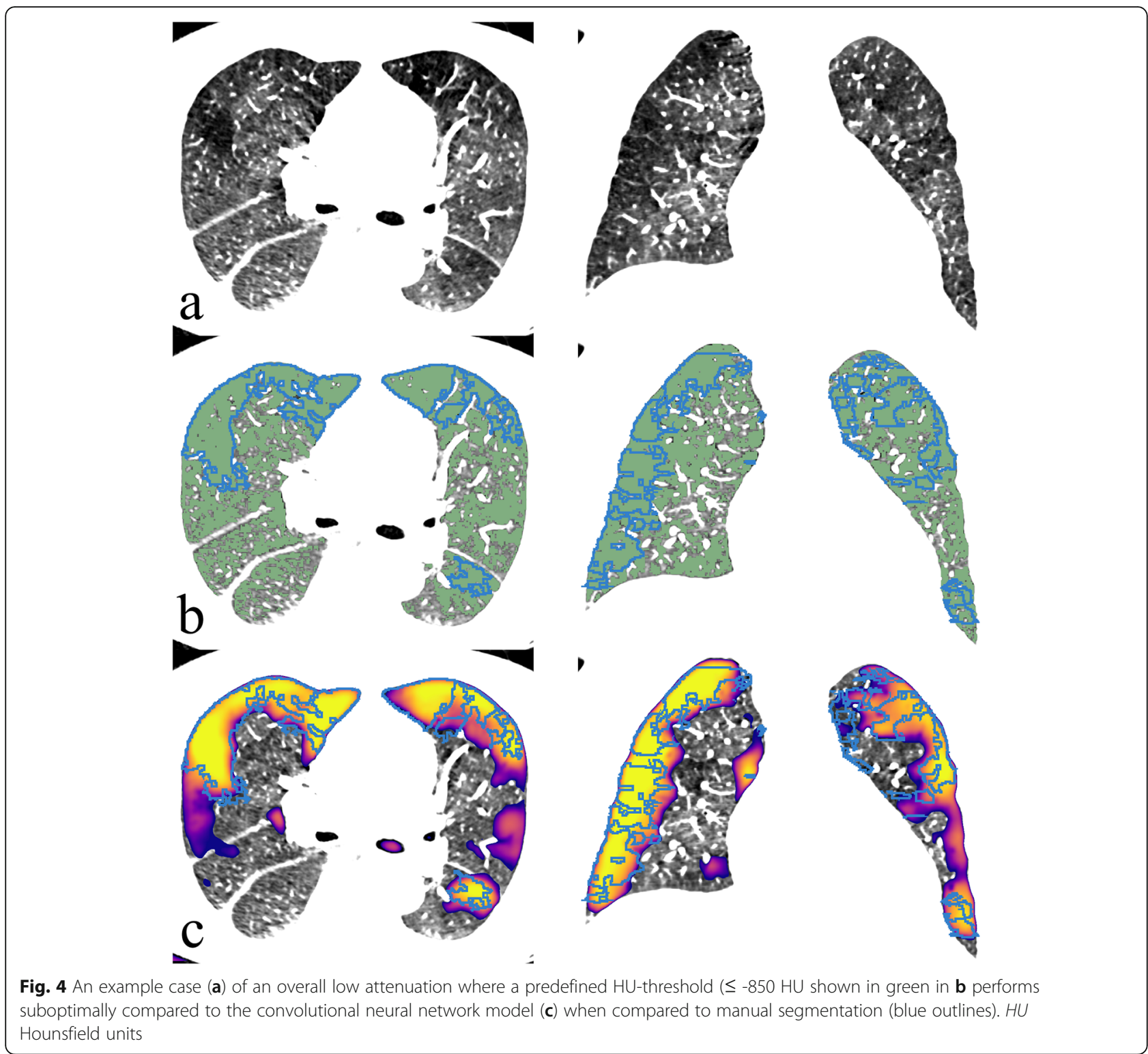

abnormalities of perfusion or aeration, which can be seen as heterogeneous lung attenuation on CT [44].

In patients with $\mathrm{CPE}$, the areas of low attenuation on CTPA represent areas of hypoperfusion [45], which is due to both the obstructing vascular impairment caused by chronic thrombus and the vasculopathy in the regions clear of the thrombus [6]. The low attenuation may also be due to air trapping in CTEPH patients relating to abnormalities in the affected regions' small airways [46]. Our study had only CTPA studies without expiration images, so we could not definitely differentiate the hypoperfusion and air trapping. However, regardless of the cause, hypoattenuation is associated with the regions affected by CTE, and, i.e., Bartalena et al. [47] showed that the hypoattenuating regions seen in CTPA correlated well with hypoperfusion seen in perfusion scintigraphy of patients with pulmonary hypertension including CTEPH.

Absence of mosaic perfusion is not exclusive for $\mathrm{CPE}$ as in some studies up to $26-45 \%$ of patients with CTEP $\mathrm{H}$ did not present mosaic perfusion at CTPA $[12,48]$. The mosaic perfusion is also not specific for CPE, and various diseases mimic this pattern [49]. Hence, this type of CNN algorithm would not be a comprehensive tool for the diagnosis or exclusion of $\mathrm{CPE}$, but it may help assess the extent of the disease and treatment planning. With a processing time well below $1 \mathrm{~min}$, the CNN model could be implemented as a low-latency clinical application in the picture archiving and communication system by overlaying the prediction on top of the images or by reporting the volume(s) of the hypoperfused regions. This type of application might also assist the 


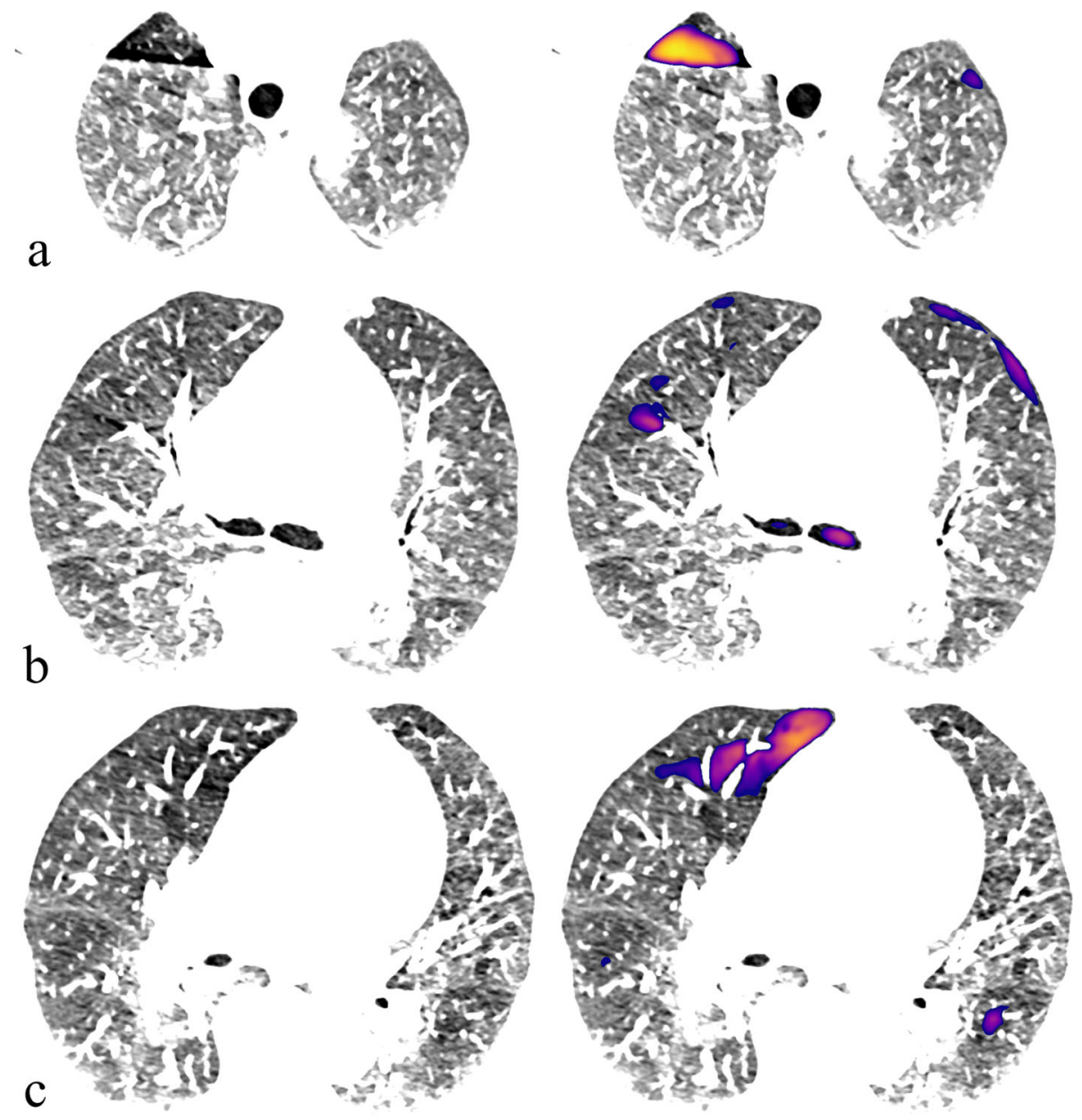

Fig. 5 Common false negative convolutional neural network predictions were related to beam hardening artefacts seen adjacent to dense contrast material in the subclavian vein (a), around vessel (b) in subpleural sites (b), and adjacent to larger organs like the heart and the hilum (c)
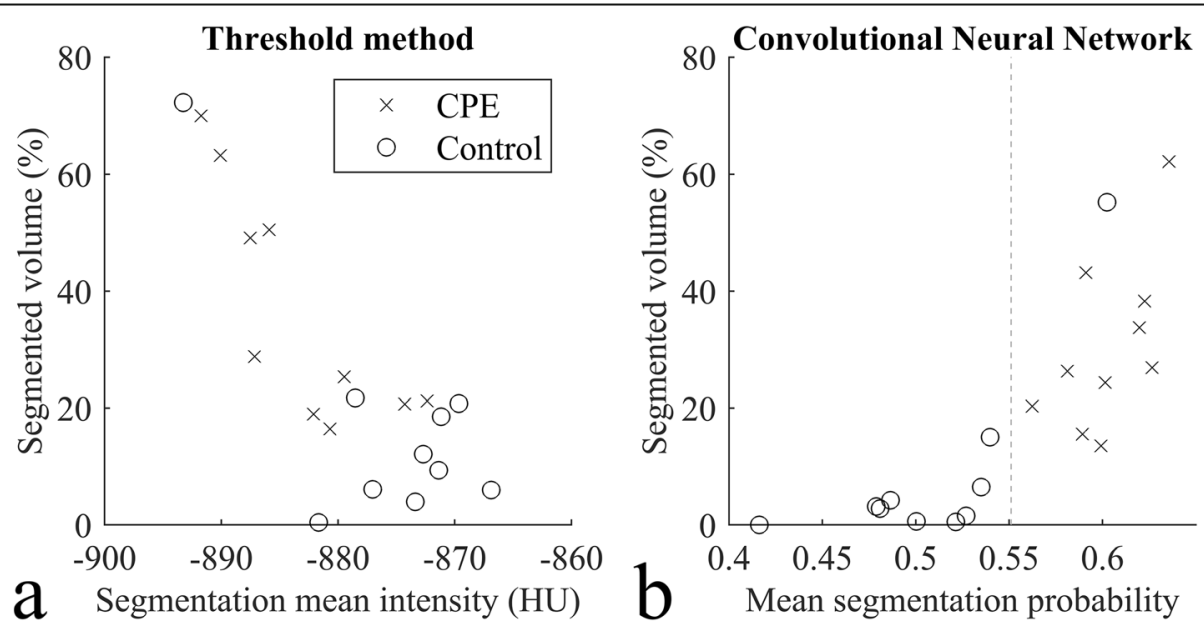

Fig. 6 CPE cases (test set cases of group A) are indicated with crosses and the controls (test set cases of group B) with circles. CPE Chronic pulmonary embolism, HU Hounsfield units 


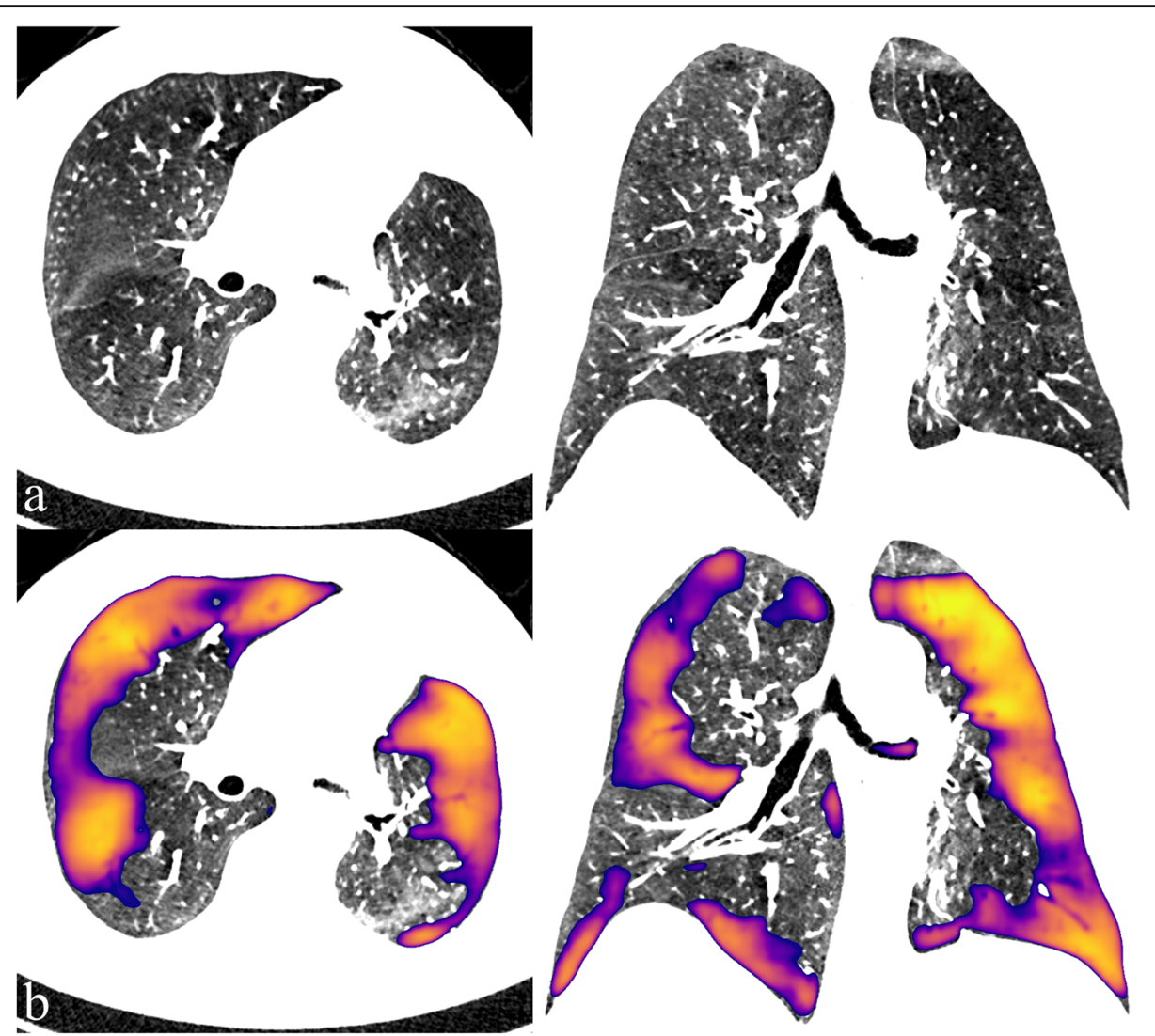

Fig. 7 One patient with previous upper lobe left pneumonectomy, and the remaining lung parenchyma was low in HU, resulting in large false positive predictions by the convolutional neural network. HU Hounsfield units

radiologist in diagnosing $\mathrm{CPE}$, which is often misdiagnosed and difficult to detect, especially if combined with an algorithm assessing the vascular defects. A more extensive database is needed to conclude the feasibility of $\mathrm{CNN}$ algorithms in a clinical setting and with automatic methods of detecting the vascular defects.

A limitation of our study was that the labels were manually segmented by a single radiologist and contained an unknown interobserver variability and individual bias level. Contributing to this label noise was the lack of expiration CT scans, and definite differentiation between hypoperfusion and air trapping was not possible. Additionally, borders between the regions with differing perfusion were gradient in many cases, and exact demarcation was not always univocal during the preprocessing, which might explain some of the overestimation and underestimation of the lesions by the $\mathrm{CNN}$ algorithm.

The patients in the study had different parenchymal changes relating to other diseases (e.g., scars, atelectasis, pleural fluid, emphysema, inflammatory lesions), and all the patients who had these types of findings extended to over two thirds of the lung volume were excluded from the study. Although this exclusion criterion presents an unknown amount of bias in the sample profile, we found it necessary. Otherwise, the manual segmentation could not have been done objectively, leaving the segmentations too imprecise. To avoid inaccurate labelling, the smallest regions of hypoperfusion and regions with undefined borders relating to noise and artefacts were left manually unlabelled. The segmentation was done correspondingly for the whole group A (divided for the training, validation and the test sets). This had an unknown effect on the results as the CNN labelled some of these small regions correctly as hypoperfusion. Still, they presented as false positives since the manual segmentation was considered the ground truth. New practical methods for improved manual segmentation and labelling the parenchymal lesions in the lungs need to be explored. One such tool is the morphological contour interpolation introduced by Zukić et al. [31], which implements a morphology-based interslice interpolation proposed by Albu et al. [32]. In our study, this tool turned out invaluable for the manual segmentation since the lesions were large in number and volume with irregular shapes and borders, and not always well demarcated in every CT slice. 
Finally, we acknowledge as a study limitation the small sample size, for which only a modest anatomical and pathological variation were available during training. Also, the small number of validation cases may have led to a suboptimal stopping point and model selection. Nevertheless, to address the resulting problem of unknown generalizability, the data portion dedicated to testing was kept relatively high in the study design. Likely, a big data approach (e.g., training with additional, more readily available, non-CPE cases) would be beneficial, especially in decreasing the extent and frequency of false-positive regions.

In conclusion, this study demonstrated the feasibility of a deep learning algorithm for the detection of hypoperfusion in CPE from CTPA with a good performance. These encouraging findings suggest that CNNs could be used as an automated method for assisting the clinician and radiologist in diagnosis and treatment planning for patients with CPE.

\section{Abbreviations}

AUC: Area under curve; Cl: Confidence interval; CPE: Chronic pulmonary embolism; CT: Computed tomography; CTED: Chronic thromboembolic disease; CTEPH: Chronic thromboembolic pulmonary hypertension; CTPA: CT pulmonary angiography; MCC: Matthews correlation coefficient; V/ Q: Ventilation/perfusion

\section{Acknowledgements}

Eero Salli, PhD (Tec.) and Ulla Wilppu, M.Sc. (Tec.) are acknowledged for their technical assistance and stimulated discussions during the project.

\section{Authors' contributions}

All the authors participated in planning the study. TV did the patient selection, clinical analysis, review, and manual segmentation of the patient cases. TM designed the pre-processing and analysis phases. The neural network workflow was implemented by TM. MK and SS gave valuable medical and technical insight throughout the project. TM and TV prepared the body of the manuscript. All the authors have improved and approved the manuscript.

\section{Funding}

This study received research and development funding from Helsinki University Hospital (SS: TYH2019253, MK: Y780020122 and TV: Y780019034).

\section{Availability of data and materials}

The trained deep learning models are available on reasonable request sent to the corresponding author.

\section{Declarations}

Ethics approval and consent to participate

Ethical committee approved this retrospective study, and patients' informed consent was waived.

\section{Consent for publication}

Not applicable.

\section{Competing interests}

The authors have no conflicts of interest to declare.

\section{Author details}

${ }^{1}$ HUS Medical Imaging Center, Radiology, University of Helsinki and Helsinki University Hospital, P.O. Box 340 (Haartmaninkatu 4), Fl-00290 Helsinki,
Finland. ${ }^{2}$ Department of Physics, University of Helsinki, P.O. Box 64, Fl-00014 Helsinki, Finland.

Received: 15 February 2021 Accepted: 26 July 2021

Published online: 24 September 2021

\section{References}

1. Lewczuk J, Piszko P, Jagas J, et al (2001) Prognostic factors in medically treated patients with chronic pulmonary embolism. Chest 119:81-823. https://doi. org/10.1378/chest.119.3.818

2. Riedel M, Stanek V, Widimsky J, Prerovsky I (1982) Longterm follow-up of patients with pulmonary thromboembolism. Late prognosis and evolution of hemodynamic and respiratory data. Chest 81:151-158. https://doi.org/1 $0.1378 /$ chest.81.2.151

3. Sanchez O, Helley D, Couchon S et al (2010) Perfusion defects after pulmonary embolism: risk factors and clinical significance. J Thromb Haemost 8:1248-1255. https://doi.org/10.1111/j.1538-7836.2010.03844.x

4. Cosmi B, Nijkeuter M, Valentino M, Huisman MV, Barozzi L, Palareti G (2011) Residual emboli on lung perfusion scan or multidetector computed tomography after a first episode of acute pulmonary embolism. Intern Emerg Med 6:521-528. https://doi.org/10.1007/s11739-011-0577-8

5. Ruggiero A, Screaton NJ (2017) Imaging of acute and chronic thromboembolic disease: state of the art. Clin Radiol 72:375-388. https://doi. org/10.1016/j.crad.2017.02.011

6. Moser KM, Bloor CM (1993) Pulmonary vascular lesions occurring in patients with chronic major vessel thromboembolic pulmonary hypertension. Chest 103:685-692. https://doi.org/10.1378/chest.103.3.685

7. Hoeper MM, Mayer E, Simonneau G, Rubin LJ (2006) Chronic thromboembolic pulmonary hypertension. Circulation 113:2011-2020. https://doi.org/10.1161/CIRCULATIONAHA.105.602565

8. Konstantinides SV, Meyer G, Becattini C, et al (2019) 2019 ESC guidelines for the diagnosis and management of acute pulmonary embolism developed in collaboration with the European Respiratory Society (ERS): the task force for the diagnosis and management of acute pulmonary embolism of the European Society of Cardiology (ESC). Eur Heart J 41:543-603. https://doi. org/10.1093/eurheartj/ehz405

9. Delcroix M, Kerr K, Fedullo P (2016) Chronic thromboembolic pulmonary hypertension. Epidemiology and risk factors. Ann Am Thorac Soc 13:S201S206. https://doi.org/10.1513/AnnalsATS.201509-621AS

10. Pepke-Zaba J, Jansa P, Kim NH, Naeije R, Simonneau G (2013) Chronic thromboembolic pulmonary hypertension: role of medical therapy. Eur Respir J 41:985-990. https://doi.org/10.1183/09031936.00201612

11. Auger WR, Kerr KM, Kim NH, Fedullo PF (2012) Evaluation of patients with chronic thromboembolic pulmonary hypertension for pulmonary endarterectomy. Pulm Circ 2:155-162. https://doi.org/10.4103/2045-8932 97594

12. Tunariu N, Gibbs SJR, Win Z, et al (2007) Ventilation-perfusion scintigraphy is more sensitive than multidetector CTPA in detecting chronic thromboembolic pulmonary disease as a treatable cause of pulmonary hypertension. J Nucl Med 48:680-684. https://doi.org/10.2967/jnumed.106. 039438

13. Keogh AM, Mayer E, Benza RL, et al (2009) Interventional and surgical modalities of treatment in pulmonary hypertension. J Am Coll Cardiol 54: S67-S77. https://doi.org/10.1016/j.jacc.2009.04.016

14. Coulden R (2006) State-of-the-art imaging techniques in chronic thromboembolic pulmonary hypertension. Proc Am Thorac Soc 3:577-583. https://doi.org/10.1513/pats.200605-119LR

15. He J, Fang W, Lv B, et al (2012) Diagnosis of chronic thromboembolic pulmonary hypertension: comparison of ventilation/perfusion scanning and multidetector computed tomography pulmonary angiography with pulmonary angiography. Nucl Med Commun 33:459-463. https://doi.org/1 0.1097/MNM.0b013e32835085d9

16. Leone MB, Giannotta M, Palazzini M, et al (2017) A new CT-score as index of hemodynamic changes in patients with chronic thromboembolic pulmonary hypertension. Radiol Med 122:495-504. https://doi.org/10.1007/ s11547-017-0750-x

17. Castañer E, Gallardo X, Ballesteros E, et al (2009) CT diagnosis of chronic pulmonary thromboembolism. Radiographics 29:31-50. https://doi.org/1 $0.1148 /$ rg.291085061

18. Mahammedi A, Oshmyansky A, Hassoun PM, Thiemann DR, Siegelman SS (2013) Pulmonary artery measurements in pulmonary hypertension: the role 
of computed tomography. J Thorac Imaging 28:96-103. https://doi.org/10.1 097/RTI.0b013e318271c2eb

19. Devaraj A, Wells AU, Meister MG, Corte TJ, Wort SJ, Hansell DM (2010) Detection of pulmonary hypertension with multidetector $C T$ and echocardiography alone and in combination. Radiology 254:609-616. https://doi.org/10.1148/radiol.09090548

20. Sugiura T, Tanabe N, Matsuura Y, et al (2013) Role of 320-slice CT imaging in the diagnostic workup of patients with chronic thromboembolic pulmonary hypertension. Chest 143:1070-1077. https://doi.org/10.1378/chest.12-0407

21. Gopalan D, Blanchard D, Auger WR (2016) Diagnostic evaluation of chronic thromboembolic pulmonary hypertension. Ann Am Thorac Soc 13:S222S239. https://doi.org/10.1513/AnnalsATS.201509-623AS

22. Rogberg AN, Gopalan D, Westerlund E, Lindholm P (2019) Do radiologists detect chronic thromboembolic disease on computed tomography? Acta Radiol 60:1576-1583. https://doi.org/10.1177/0284185119836232

23. Fedullo PF, Auger WR, Kerr KM, Rubin LJ (2001) Chronic thromboembolic pulmonary hypertension. N Engl J Med. 345:1465-1472. https://doi.org/10.1 056/NEJMra010902

24. Pepke-Zaba J, Delcroix M, Lang I, et al (2011) Chronic thromboembolic pulmonary hypertension (CTEPH): results from an international prospective registry. Circulation 124:1973-1981. https://doi.org/10.1161/CIRCULATIONA HA.110.015008

25. Wittenberg R, Peters JF, Sonnemans JJ, Prokop M, Schaefer-Prokop C (2010) Computer-assisted detection of pulmonary embolism: evaluation of pulmonary CT angiograms performed in an on-call setting. Eur Radiol 20: 801-806. https://doi.org/10.1007/s00330-009-1628-7

26. Chan H, Hadjiiski L, Zhou C, Sahiner B (2008) Computer-aided diagnosis of lung cancer and pulmonary embolism in computed tomography-a review. Acad Radiol 15:535-555. https://doi.org/10.1016/.jacra.2008.01.014

27. Galiè N, Humbert M, Vachiery J et al (2015) 2015 ESC/ERS guidelines for the diagnosis and treatment of pulmonary hypertension. Eur Respir J 46:903975. https://doi.org/10.1183/13993003.01032-2015

28. Taboada D, Pepke-Zaba J, Jenkins DP, et al (2014) Outcome of pulmonary endarterectomy in symptomatic chronic thromboembolic disease. Eur Respir J 44:1635-1645. https://doi.org/10.1183/09031936.00050114

29. Marcus DS, Olsen TR, Ramaratnam M, Buckner RL (2007) The extensible neuroimaging archive toolkit: an informatics platform for managing, exploring, and sharing neuroimaging data. Neuroinformatics 5:11-34. https://doi.org/10.1385/ni:5:1:11

30. Fedorov A, Beichel R, Kalpathy-Cramer J, et al (2012) 3D slicer as an image computing platform for the quantitative imaging network. Magn Reson Imaging 30:1323-1341. https://doi.org/10.1016/j.mri.2012.05.001

31. Zukić D, Vicory J, McCormick M et al (2016) ND morphological contour interpolation. In: Insight Journal. http://hdl.handle.net/10380/3563. Accessed 9 Aug 2016

32. Albu A, Beugeling T, Laurendeau D (2008) A morphology-based approach for interslice interpolation of anatomical slices from volumetric images. IEEE Trans Biomed Eng 55:2022-2038. https://doi.org/10.1109/TBME.2008.921158

33. Ronneberger O, Fischer P, Brox T (2015) U-Net: Convolutional networks for biomedical image segmentation. In: Navab N, Hornegger J, Wells WM, Frangi AF (eds) MICCAI 2015: 18th international conference on medical image computing and computer-assisted intervention, Munich, October 2015, Lecture notes in computer science, vol 9351. Springer, Cham, pp 234241. https://doi.org/10.1007/978-3-319-24574-4_28

34. Abadi M, Barham P, Chen J, Chen Z, Davis A, Dean J (2016) TensorFlow: a system for large-scale machine learning. In: Proceedings of the 12th USENIX conference on operating systems design and implementation (OSDI'16), Savannah, 2-4 November 2016

35. Kingma D, Ba J (2015) Adam: a method for stochastic optimization. In: Proceedings of the 3rd International Conference on Learning Representations (ICLR 2015), San Diego, 7-9 May 2015

36. Milletari F, Navab N, Ahmadi S (2016) V-Net: Fully convolutional neural networks for volumetric medical image segmentation. In: Proceedings of the 2016 4th international conference on 3D vision (3DV), Stanford, 25-28 October 2016

37. Chicco D, Jurman G (2020) The advantages of the Matthews correlation coefficient (MCC) over F1 score and accuracy in binary classification evaluation. BMC Genomics 21:6. https://doi.org/10.1186/s12864-019-6413-7

38. Nemec SF, Bankier AA, Eisenberg RL (2013) Pulmonary hyperlucency in adults. AJR Am J Roentgenol 200:W101-W115. https://doi.org/10.2214/A JR.12.8917
39. Huang S, Kothari T, Banerjee I et al (2020) PENet-a scalable deep-learning model for automated diagnosis of pulmonary embolism using volumetric CT imaging. NPJ Digit Med 3:61. https://doi.org/10.1038/s41746-020-0266-y

40. Tajbakhsh N, Gotway M, Liang J (2015) Computer-aided pulmonary embolism detection using a novel vessel-aligned multi-planar image representation and convolutional neural networks. In: MICCAI 2015: 18th international conference on medical image computing and computerassisted intervention, Munich (October 2015) Lecture notes in computer science, vol 9350. Springer, Cham, pp 62-69

41. Yang X, Lin Y, Su J, et al (2019) A two-stage convolutional neural network for pulmonary embolism detection from CTPA images. IEEE Access 7:8484984857. https://doi.org/10.1109/ACCESS.2019.2925210

42. Liu W, Liu M, Guo X, et al (2020) Evaluation of acute pulmonary embolism and clot burden on CTPA with deep learning. Eur Radiol 30:3567-3575. https://doi.org/10.1007/s00330-020-06699-8

43. Öman O, Mäkelä T, Salli E, Savolainen S, Kangasniemi M (2019) 3D convolutional neural networks applied to CT angiography in the detection of acute ischemic stroke. Eur Radiol Exp 3:8. https://doi.org/10.1186/s41747019-0085-6

44. Hansell DM (2010) Thin-section CT of the lungs: the hinterland of normal. Radiology 256:695-711. https://doi.org/10.1148/radiol.10092307

45. Goerne H, Batra K, Rajiah P (2018) Imaging of pulmonary hypertension: an update. Cardiovasc Diagn Ther 8:279-296. https://doi.org/10.21037/cdt.2018. 01.10

46. Arakawa H, Stern EJ, Nakamoto T, Fujioka M, Kaneko N, Harasawa H (2003) Chronic pulmonary thromboembolism. Air trapping on computed tomography and correlation with pulmonary function tests. J Comput Assist Tomogr 27:735-742. https://doi.org/10.1097/00004728-200309000-00010

47. Bartalena T, Oboldi D, Guidalotti PL, et al (2008) Lung perfusion in patients with pulmonary hypertension: comparison between MDCT pulmonary angiography with minIP reconstructions and 99mTc-MAA perfusion scan. Invest Radiol 43:368-373. https://doi.org/10.1097/RLI.0b013e31816901e2

48. Sherrick AD, Swensen SJ, Hartman TE (1997) Mosaic pattern of lung attenuation on $\mathrm{CT}$ scans: frequency among patients with pulmonary artery hypertension of different causes. AJR Am J Roentgenol 169:79-82. https:// doi.org/10.2214/ajr.169.1.9207504

49. Kligerman SJ, Henry T, Lin CT, Franks TJ, Galvin JR (2015) Mosaic attenuation: etiology, methods of differentiation, and pitfalls. Radiographics 35:13601380. https://doi.org/10.1148/rg.2015140308

\section{Publisher's Note}

Springer Nature remains neutral with regard to jurisdictional claims in published maps and institutional affiliations.

\section{Submit your manuscript to a SpringerOpen ${ }^{\circ}$ journal and benefit from:}

- Convenient online submission

- Rigorous peer review

- Open access: articles freely available online

- High visibility within the field

- Retaining the copyright to your article

Submit your next manuscript at $\boldsymbol{\nabla}$ springeropen.com 